\title{
PSICOTERAPIA ON LINE \\ Escenario virtual para una relación real en el espacio intermediario
}

\author{
Victor Cabré y Josep Mercadal ${ }^{1}$ \\ Fundació Vidal i Barraquer, Barcelona
}

Los numerosos e importantes cambios sociales protagonizados por internet y el fenómeno on line, están provocando que la forma de comunicarnos, de relacionarnos y de establecer vínculos, no se parezca en nada a la de hace solo unos años atrás. Estas transformaciones han llegado a todos los estamentos de nuestra sociedad y tanto es así que desde la práctica clínica psicológica cada vez son más las demandas de tratamientos on line. Ésta, cómo otras tantas novedades que han aparecido a lo largo de la historia de la psicología, no debería incorporarse en el marco de trabajo sin analizar previamente cuáles pueden ser sus efectos. El presente trabajo reflexiona acerca de los elementos que, a partir de nuestra práctica clínica, pensamos que deben de tenerse especialmente en cuenta antes de empezar un tratamiento on line. La transferencia, el encuadre y la alianza terapéutica, son solo algunos de los aspectos que analizaremos, enfatizando aquellos en los que hemos detectado las diferencias más significativas entre la terapia presencial y la on line. Al no tratarse de dos tipos distintos de terapia sino de la utilización intencionada de una herramienta comunicativa diferente, se postulan las principales características con el objetivo de que no se vea afectado ni el correcto desarrollo del tratamiento ni su eficacia.

Palabras clave: Psicoterapia on line, psicoterapia por internet, comunicación virtual, espacio intermediario, psicoanálisis relacional.

The numerous and important social changes produced by Internet and the on line phenomenon makes a big difference in how we relate, communicate and link, than not so long ago. These transformations have arrived to entire states of our society in such a way that in the clinical psychology are more and more demands for on line treatment. This, like many other innovations that have appeared throughout the history of psychology, should not be incorporated to the work setting without first analyzing which might be the effects. This paper reflects on the elements that, from our clinical experience, we can think over and can take into account or consider before starting an on line treatment. The transfer, setting, and therapeutic alliance, are just some of the aspects that we are going to analyze, emphasizing those that we detected as the most significant differences between in-person and on line therapy. Since it is not about two different types of therapy but about the intentional use of a different communicative tool, we postulated the main features with the aim that it doesn't concern neither the proper development of the treatment nor its effectiveness.

Key Words: On line Psychotherapy, Psychotherapy by internet, virtual communication, intermediate space, relational psychoanalysis.

English Title: ON LINE PSYCHOTHERAPY. Virtual stage for a real relationship in the intermediate space.

Cita bibliográfica / Reference citation:

Cabré, V. y Mercadal, J. (2016). Psicoterapia On-line. Escenario Virtual para una relación real en el espacio intermediario. Clínica e Investigación Relacional, 10 (2): 439-452. [ISSN 1988-2939]

[Recuperado de www.ceir.info ] DOI: 10.21110/19882939.2016.100206

${ }^{1}$ Correspondencia: vcabre@fvb.cat; jmercadal@fvb.cat. Fundació Vidal i Barraquer. c/Sant Gervasi de Cassoles 88-90, 08022 Barcelona 


\section{Introducción}

Resulta obvio decir que, en las últimas décadas, el ámbito de la comunicación es en el que se han producido los cambios más veloces y radicales. Los abuelos de hoy, durante su juventud, se comunicaban entre ellos mediante cartas. Podían pasar meses antes de recibir respuesta de su amado, familiar o amigo. Más adelante, el teléfono permitió una comunicación más rápida, facilitando la representación mental del otro a través de la voz en directo. Hoy en día, tardamos un "clic" en poder ver en directo a quién queramos por distante que esté geográficamente. Así, las modalidades de comunicación humana, las relaciones intersubjetivas y las representaciones que tenemos de nuestra relación con los otros y con nosotros mismos se han transformado radicalmente (Vaimberg, 2014).

El mundo, en su totalidad, se ha visto impregnado y condicionado por el nuevo gigante de la comunicación. Tanto es así que, en muchos aspectos, podría parecer que poco queda del cogito ergo sum ("pienso, luego existo") de Descartes, que se ha visto transformado en "estoy en la red, luego existo", con lo que, quién pretenda vivir al margen de las nuevas tecnologías de la comunicación, seguramente está destinado a vivir fuera de la sociedad. Las pantallas son ya parte imprescindible de nuestra vida y buena parte de ella se desarrolla en diferentes pantallas, cambiando o creando nuevas maneras de relacionarse, pensar o vivir nuestra propia identidad (Vaimberg, 2012). En este sentido, Bauman (2005) propone el concepto de Sociedad Líquida con el que se pregunta, entre otras cosas, si esta forma de relación humana, basada en conexiones, podrá llenar el vacío que dejan los "antiguos" vínculos. A nuestro entender y siguiendo a Baudrillard (2005), este cambio basado en las conexiones no es más que una simulación, la imagen pasa de ser una representación de la realidad a convertirse en su propio simulacro, dejando así, enterrada la realidad.

Es tal la magnitud de este fenómeno, que son pocos los ámbitos que no se han visto invadidos, amenazados, condicionados o, cuanto menos, influenciados por él. La psicología, desde luego, no es uno de ellos. En la práctica clínica hemos observado que, durante los últimos años, han aumentado de forma exponencial las demandas de iniciar una terapia on line. Este hecho, sin duda, conlleva una serie de cambios que no podemos obviar y frente los cuales proponemos dos consideraciones previas:

En primer lugar, hay que tener en cuenta que, propiamente dicho, no estamos hablando de una nueva modalidad de intervención terapéutica, sino de la utilización de un recurso técnico particular adaptado a cualquiera de las modalidades descritas y manualizadas de psicoterapia. Aún así, es evidente que cualquier nuevo elemento técnico introduce cambios 
que hay que comprender, sistematizar y gestionar, para que la modalidad terapéutica utilizada siga siendo, al menos, igual de eficaz que lo habría sido sin la incorporación de dicha novedad.

Una segunda premisa es la de que, habitualmente, no es el profesional el que introduce este elemento técnico como una forma de mejorar los resultados del trabajo que está realizando con el paciente. Por lo general, nos referiremos aquí al uso del ordenador como una forma de resolver circunstancias que dificultan o impiden la comunicación presencial entre terapeuta y paciente como primera opción, aunque no descartamos que, en un futuro, su utilización pueda incrementarse notablemente y hasta pueda convertirse en habitual.

Lo que nos interesa en el presente trabajo es hacer un análisis de cómo las nuevas tecnologías de la comunicación influyen en las terapias psicoanalíticas o, lo que es lo mismo, qué papel juegan las psicoterapias on line y cómo deben usarse. No nos centraremos en la utilización ocasional de estos elementos técnicos, sino cuando su uso es habitual en el desarrollo de una relación terapéutica. Es evidente su utilidad para establecer un contacto puntual con el paciente en situaciones urgentes que demandan inmediatez, o en momentos determinados de un proceso terapéutico que requieren versatilidad por parte del profesional, o en un período de mantenimiento una vez finalizada la intervención terapéutica. Todas estas situaciones y muchas más son, actualmente, de uso corriente y no precisan mayor atención que la que otorgamos a otros elementos técnicos, tanto en lo que se refiere a consideraciones éticas, de encuadre, de contrato terapéutico, de disponibilidad del terapeuta, de condiciones de trabajo o de formación del profesional. Cuando el formato on line se convierte en el vehículo principal de la comunicación y de la relación terapéutica, creemos que se hace necesaria la reflexión de hasta qué punto se ven modificadas o no las condiciones estudiadas y sistematizadas por la investigación en psicoterapia.

Para muchos autores no existe una definición establecida de lo que se entiende como psicoterapia on line. Ya en 1998, Brown definía la Telepsiquiatría como el uso de la tecnología de las telecomunicaciones para relacionar a los pacientes con los profesionales. Se proponía facilitar el diagnóstico, la educación, las consultas, el tratamiento, el almacenamiento y la transmisión de datos médicos del paciente a los profesionales, investigadores y otras actividades relacionadas con la salud mental. Nickelson (1998), definió Telesalud como el uso de las telecomunicaciones y la tecnología de la información para proveer el acceso a la evaluación de la salud, diagnóstico, intervención, consulta, supervisión, educación e información a distancia. Ambas propuestas, por tanto, incluyen múltiples avances 
tecnológicos en materia de comunicación que van, desde el teléfono y el fax, hasta el correo electrónico y las diversas modalidades de chat.

Más recientemente, Rochlen, Zack y Speyer (2004) afirmaban que se trata de cualquier tipo de interacción profesional, de índole terapéutica, que toma el uso de internet como medio para la práctica en salud mental. Para Botella y otros (2007), el concepto de psicoterapia on line deviene algo más amplio, y prefieren denominarlo, en el mismo sentido que Brown, Telepsicología. En 2012, Vaimberg expone que las psicoterapias o intervenciones mediadas tecnológicamente (PMT) son aquellas que, respondiendo a los objetivos de las psicoterapias o de las diferentes intervenciones en salud mental, lo hacen utilizando las Tecnologías de la Información y la Comunicación (TIC) y afirma, además, que son los pacientes los que nos han ido llevando por este apasionante camino, enseñándonos lo que había que hacer, cómo y cuándo.

En cualquier caso, pensamos que el término con el que denominamos la práctica psicoterapéutica a través de internet no es la cuestión más importante a resolver, ni tampoco su propia existencia, ya que parece que esta herramienta psicoterapéutica ha llegado para quedarse. Autores como Anderson, Carlbring, Berger, Almlöv y Cuijpers (2009) afirman que la psicoterapia on line (así la llamaremos nosotros) es un tipo de terapia psicológica que funciona, pero la investigación aún no ha conseguido determinar qué hace que funcione, al igual que tampoco ha conseguido determinar indicadores de resultados.

\section{El formato On line}

Internet cuenta con distintos medios para establecer la comunicación entre paciente y terapeuta. Aunque autores como Wright (2002) destaquen los efectos terapéuticos de la escritura y propongan un tipo de psicoterapia mediante el correo electrónico o el chat, nosotros entendemos la psicoterapia on line a través de la videoconferencia, ya que permite una interacción con un alto grado de componentes sensoriales (Castelnuovo, Gaggioli, Mantovani y Riva, 2003; Pergament, 1998) homologándose, así, a la tradicional psicoterapia presencial. Antes de empezar una terapia on line, pensamos que deben hacerse presentes las circunstancias que han llevado a iniciar este tipo de terapia y no otra, mediante consideraciones como las siguientes: ¿es lo mismo empezar una terapia directamente vía internet, sin conocer al paciente y accediendo a su petición de terapia on line? o por el contrario, si se está llevando a cabo una terapia presencial pero, por motivos ajenos a la terapia, no se puede proseguir debido a que el paciente o el terapeuta abandonan la ciudad, 
¿es indistinto que sea el paciente o el terapeuta el que abandone el marco de trabajo inicialmente previsto? o ¿estará siempre indicado que una terapia tenga continuidad a través de internet?.

Pensamos que lo primero que hay que destacar es la soledad del terapeuta. El terapeuta se enfrenta a una visita con el despacho vacío. En nuestro equipo trabajan terapeutas que llevan muchos años cuidando de una serie de aspectos del encuadre considerados fundamentales para el buen hacer de la terapia y al introducir en la terapia el componente on line, éste setting que durante tantos años ha prevalecido en nuestra práctica clínica se ve alterado hasta el punto de sacar al paciente del despacho. Éste hecho puede privar al terapeuta de una serie de datos sobre la comunicación no verbal, el aspecto físico completo del paciente, la percepción de los olores, la distorsión de la voz o el modo y con quién acude el paciente a la sesión, pero por otro lado puede permitirle penetrar en su domicilio o lugar elegido para llevar a cabo las sesiones. Veamos pues, cómo estos elementos influyen, modifican o cambian la manera de trabajar.

Muchos son los autores (Encabo y López, 2004; Cestero, 2006; Valdés, Krause y Álamo, 2011; García, Segura, Palanca y Román, 2012) que destacan la superioridad que tienen los aspectos no verbales respecto a los verbales en la oratoria comunicativa. Tanto es así que se considera que en una conversación, el predominio de los componentes verbales está alrededor del 35\%, mientras que, los no verbales, se acercan al 65\% (Nuñez-Delgado, 2000; Tusón, 2002; Poyatos, 2003). Ante estos datos no podemos obviar la pérdida que supone para la relación y la comunicación entre paciente y terapeuta, el paso de una terapia presencial a una on line.

Un elemento de la comunicación no verbal es el aspecto físico. Evidentemente, mediante internet no podemos percibir los olores, así como tampoco podemos elegir qué vemos del paciente ya que será él quien decida enseñarnos algunas cosas y esconder otras. De este modo, perdemos la ocasión de introducir en la terapia aspectos de su vida cotidiana, un olor que nos haga deducir que viene de hacer alguna actividad concreta, un aspecto físico especialmente cuidado o descuidado, o cualquier otra cosa que quizás nos interese introducir en el marco de trabajo.

La voz es otro de estos aspectos no verbales que también aportan información: el tono, la fuerza, la potencia o la fluidez son elementos que nos ayudan a intuir un trasfondo en lo que nos está contando el paciente. Estos aspectos evidentemente se mantienen en una terapia on line, pero nuestra experiencia nos dice que no los percibimos igual. Cómo terapeutas, nos hemos descubierto alzando más la voz y pronunciando frases más cortas y sintéticas en 
sesiones on line que presenciales, en un intento por salvar la distancia física proyectando la voz y simplificando la construcción de las frases, y es que ya no sólo depende de cómo nosotros digamos las cosas y el otro las reciba, sino también de cómo el ordenador las recibe en nuestro despacho y las emite al paciente, y viceversa. Hemos de tener en cuenta, además, que a través del ordenador el sonido de la voz es más metálico, menos susceptible de ser amortiguado por los sistemas habituales de aislamiento en paredes y puertas de la mayor parte de despachos profesionales (lo que conlleva otras implicaciones de privacidad y de contaminación acústica), pero sobretodo dificultando la modulación de la voz y la percepción de sus matices. Así pues, la incorporación del ordenador a la sesión, supone una serie de cambios obvios y previstos, pero a la vez fuerza al paciente y al terapeuta a hacer cambios en su discurso, en su forma de comunicarse, cambios que no se habían podido intuir y que muchas veces se mantienen en el inconsciente y no son analizados. El hecho de hacer frases más escuetas y sintéticas, no es sino un modo de explotar la comunicación verbal ya que, como hemos comentado, la no verbal se ve mermada. Así, cuando en la visita se podían decir cosas más lentamente, pensando, gesticulando, dejando espacios y silencios que ambos, paciente y terapeuta, podían vivir de la misma manera, ahora, con el artefacto mediador incorporado en la sesión, se abren interrogantes entre paciente y terapeuta acerca de lo que percibirá el otro ante un gesto, una mirada, una pausa o un silencio, y como diría Vaimberg (2012), la pantalla del ordenador se erige como un espacio transicional que dará lugar a una diversidad de proyecciones determinadas por las características de personalidad de los participantes conectados así como por las características de las interacciones, optándose por articular frases cortas y concisas que se puedan procesar rápidamente y que dejen menor espacio para elementos no verbales. De este modo, la terapia on line se convierte en una terapia dialéctica en la que los elementos quizás más "cálidos" como son las miradas, los silencios en momentos delicados o los gestos sutiles, se van perdiendo o quedando en un segundo plano y, con ellos, los elementos más relacionales y emocionales, quedando bajo el amparo de lo racional y tal vez teórico.

Con los silencios ocurre algo parecido a los sonidos, ya que el ordenador no se limita a reproducirlos fielmente, sino que parece como si adquirieran nuevas connotaciones, como si los silencios fueran más largos, o más vacíos, o más densos, etc. La revolución digital ha fracasado completamente en la reproducción del silencio ya que, a diferencia de lo que ocurría con su antecesor analógico, ha conseguido un vacío absoluto de sonido que raramente existe en la naturaleza "sonando" artificioso y poco real.

Con respecto a la imagen, podríamos decir que el despacho se transforma en una pantalla, donde lo que hay alrededor de ésta queda mucho más en un segundo plano y cada uno de los 
protagonistas sólo ve una parte del otro, tal vez simbolizando lo que en realidad ocurre en cualquier relación terapéutica: que en realidad sólo acabamos conociendo una pequeña parte de la vida del paciente y él de la nuestra. A pesar de las características del aparato y de las buenas condiciones de la conexión, la imagen siempre es menos definida que la percibida presencialmente, está más pixelada y en el mejor de los casos son inevitables las pequeñas interferencias, además de las obvias limitaciones que supone la falta de profundidad dimensional. Una vez más la pantalla no permite los matices del gesto, de la expresión facial y todavía tiene otra particularidad, como lo es el hecho de que la imagen de la cara acostumbra a verse más cercana que en la disposición presencial pero, a diferencia de lo que ocurre en ésta, la disposición de las cámaras respectivas conlleva que, prácticamente en ningún momento, los participantes se miren directamente a los ojos, ya que cuando uno lo hace el otro recibe esa mirada apuntando a la altura del pecho, apareciendo la paradoja de que la proximidad aumenta "la distancia" entre dos personas que se comunican sin apenas "mirarse a los ojos".

Cabe señalar, también, la pérdida de claves visuales y auditivas, y los problemas que ésta conlleva a la hora de gestionar la información que un paciente revela sobre su identidad (Rochlen, Zack y Speyer, 2004; Trujillo Borrego, 2005; Vallejo y Jordán, 2007), así como las dificultades que implican el manejo de situaciones de crisis y la falta de formación de los profesionales en estas nuevas formas de psicoterapia (Rochlen, Zack y Speyer, 2004; Trujillo Borrego, 2005).

\section{Algunos aspectos sobre la Transferencia}

En principio podemos afirmar que tanto la transferencia como la contratransferencia, elementos cruciales para la comprensión de la psicoterapia psicoanalítica, presentarán las mismas características en ambas modalidades de psicoterapia: la presencial y la on line. El paciente traslada pautas y modelos de sus relaciones más significativas a la relación con el profesional y éste reacciona emocionalmente a la comunicación que le llega de él. Respecto de la forma en la que se presentan podría parecer, por lo dicho hasta ahora, que ambas estarán presentes aquí de forma amortiguada, con menor intensidad y viveza (como la imagen y el sonido o como la comunicación misma) y probablemente sea así.

Con la incorporación de las nuevas tecnologías a la vida diaria parece claro que las relaciones, también las más significativas, comparten simultáneamente elementos de presencialidad y de virtualidad. La comodidad, la inmediatez y las condiciones geográficas impulsan a que, 
cada vez más, una buena parte de las relaciones familiares, amorosas o amicales tengan momentos de virtualidad a través de la pantalla. Este hecho nos pone sobre la pista de un aspecto que, a nuestro parecer, es muy relevante: la diferencia entre una relación que ya tiene un recorrido presencial antes de convertirse en on line y la relación que, ya desde sus inicios, se ha construido a través de la pantalla. Pensamos que, en psicoterapia, es preferible que los primeros contactos sean presenciales, ya que algunos aspectos de la relación transferencial y contratransferencial quedan incorporados en la mente de ambos integrantes para que progresivamente puedan transformarse a lo largo de la relación terapéutica. Luego, en la medida de lo posible, pueden acordarse sesiones presenciales ocasionales en el transcurso del proceso para, preferentemente, realizar las últimas sesiones del tratamiento de nuevo presencialmente.

Como tantos otros aspectos del encuadre, también ésta alternancia conviene que esté establecida previamente o, en cualquier caso, que sea el resultado del acuerdo entre paciente y terapeuta. De otro modo, tanto la presencia en el despacho como la distancia a través de la pantalla, pueden convertirse fácilmente en instrumentos al servicio de las resistencias y del acting out que dificulten la adquisición de comprensión y el incremento de la capacidad de insight. Obviamente es distinto si el paciente demanda las sesiones on line debido a que tiene que ausentarse por motivos de trabajo o salud, por ejemplo, que si es el terapeuta quien provoca esta nueva situación, pero en cualquier caso se hace imprescindible una cuidadosa valoración de la indicación inicial. Se podría hablar de que, en ambos casos, se incumple el contrato terapéutico inicial y que, por tanto, conviene rehacerlo o modificarlo para que vuelva a ser aceptado mutuamente y no constituya una variable extraña para el proceso. En la misma línea de significado estaría el hecho de que el paciente solicite o decida conectarse desde otro lugar distinto al habitual. Inicialmente ambos han acordado un espacio adecuado, sobretodo en términos de intimidad y privacidad: el despacho en el caso del terapeuta y una habitación a salvo de interrupciones en el caso del paciente, pero ahora éste es un aspecto del setting que el profesional "controla" menos, que en cierta medida ha delegado en el paciente.

Finalmente pensamos, con Vaimberg (2012), que el ordenador representa aquí, un verdadero espacio transicional entre terapeuta y paciente, actuando como mediador pasivo (pero distorsionador) entre ambos e incluso entre aspectos de la realidad interna y externa de ambos. Ahora, el reto es el de conseguir que este espacio sea conocido y sentido como seguro, para que pueda ser poblado de objetos transicionales que habiten la relación terapéutica y contribuyan a paliar la distancia y la no presencia. Se trataría pues de un espacio que, al mismo tiempo, acerca a los dos integrantes de la relación pero que también los aleja, que facilita el contacto con aspectos de la realidad interna pero que igualmente los dificulta, 
en esa eterna paradoja que describe Winnicott y que aconseja no intentar resolver. Tal vez la investigación en psicoterapia on line sea una buena ocasión para estudiar y revisitar el espacio transicional, en tanto que metáfora en la que tiene lugar el encuentro entre dos realidades intersubjetivas, la del paciente y la del terapeuta, en la que ambos juegan juntos en la construcción de la relación terapéutica.

\section{Notas acerca de la Alianza Terapéutica}

Otro elemento imprescindible en una psicoterapia es la alianza terapéutica. Algunos investigadores afirman que la alianza terapéutica, en las psicoterapias on line, no presenta diferencias significativas con la terapia presencial (Cook y Doyle, 2002; Hanley, 2009; Hanley y Reynolds, 2009; King, Bambling, Reid y Thomas, 2006; Knaevelsrud y Maercker, 2006; Reynolds, Stiles y Grohol, 2006), aunque a nuestro entender, es bastante improbable que una alianza terapéutica establecida desde el inicio vía on line pueda ser de la misma calidad que la presencial. Hemos destacado, hasta ahora, algunos de los aspectos diferenciales entre estos dos formatos de terapia, aunque éstos no necesariamente reflejen siempre cambios en la calidad de los mismos. En una terapia presencial que se transforma en on line, probablemente los cambios que ello supone tienen un impacto más amortiguado en lo relativo a la alianza terapéutica. Si tenemos ocasión de conocer a la persona presencialmente y establecemos con ella un vínculo o una alianza, cuando más adelante tengamos que transformar esta relación en una on line, los aspectos descritos anteriormente resultarán menos ambiguos: si hemos vivido el silencio de un paciente presencialmente, seguramente nos resultará más fácil entenderlo cuando se repita en formato on line; si hemos escuchado su voz presencialmente, seguramente nos será más fácil reconocer los aspectos no verbales (entonación, fluidez, etc.) que se nos presenten via on line. Con la expresión facial ocurrirá algo parecido: si hemos conocido al otro en persona y hemos detectado algunos patrones faciales de respuesta, será más fácil que los identifiquemos vía on line. Cuando paciente y terapeuta comparten despacho, el aire que respiran es el mismo y comparten los mismos sonidos y silencios ambientales, la misma humedad y temperatura, así como también el mismo instante horario, cosa que frecuentemente no ocurre cuando la sesión tiene lugar en la intersección de dos "despachos" distintos: el del profesional y el del paciente.

En la literatura científica, una de las principales ventajas que hemos encontrado es la de que la psicoterapia on line facilita el tratamiento a aquellas personas que viven en zonas poco urbanizadas y con escasez de recursos y profesionales (Suler, 2000; Tate y Zabinski, 2004). Pensamos entonces, que el formato on line puede facilitar el contacto con este tipo de 
pacientes, aunque ello no presupone que esta modalidad de terapia sea la más indicada. En aquellos casos en los que la única posibilidad que nos ofrece el paciente es la vía on line, hemos de intentar que la alianza sea lo más cercana posible a la primera indicación, que es la alianza establecida presencialmente. En nuestro centro hemos atendido a un gran número de pacientes que se han desplazado desde lugares alejados y nuestro proceder siempre ha priorizado la relación presencial (si es viable y no implica un sacrificio excesivo), la derivación a otro servicio o profesional como segunda opción y finalmente ofrecer la posibilidad de realizar la psicoterapia on line.

Otros autores afirman que a través de la psicoterapia on line, muchas personas que tienen dificultades en pedir la ayuda tradicional, les será beneficioso, ya que el ordenador conlleva anonimato y sensación de refugio e invisibilidad, que conducen a un sentimiento subjetivo de protección, con lo que el anonimato psicológico, posibilita compartir con un extraño temas muy personales con mayor facilidad de lo que sería en un encuentro cara a cara (Schultze, 2006; Tate y Zabinski, 2004; Vallejo y Jordán, 2007). Es comprensible que algunos pacientes puedan solicitar un trato on line para guardar distancia o sentirse más protegidos y que tal vez conviene no oponerse a esa petición, pero consideramos imprescindible trabajar sobre este foco ya que muy probablemente, detrás de esta demanda, se escondan problemas relacionales, que son los mismos que le impiden acudir presencialmente a la consulta. Si no se trabaja esta demanda, cabe el riesgo de que el terapeuta pueda aliarse con el síntoma de forma inconsciente, llegando incluso a una auténtica colusión con el paciente al servicio de sus defensas, pero con un trabajo riguroso el terapeuta puede aprovechar las facilidades que inicialmente ha supuesto ese "anonimato" para revertirlas en beneficio de una adecuada alianza terapéutica.

\section{Reflexiones finales provisionales}

Los estudios realizados hasta ahora desvelan que las intervenciones mixtas son las que presentan una mayor eficacia y que la combinación de presencialidad y virtualidad, contribuye a que el paciente sea constante en el tratamiento y, por lo tanto, a la efectividad del proceso de intervención (Ritterband et al., 2003; Griffits et al., 2006), pero lógicamente los trabajos de investigación son todavía escasos y habrá que estar atentos a como se desarrollen en el futuro.

En relación a los interrogantes que planteábamos inicialmente, creemos que la primera indicación terapéutica es la atención presencial, aunque también somos conscientes de que pueden actuar multitud de circunstancias que fuercen a cambiar los planes y tener que buscar 
alternativas. Pensamos que se puede iniciar un proceso exploratorio a un paciente que pida hacerlo vía on line, aun viviendo a pocos minutos del centro asistencial y sin ningún problema aparente que le impida desplazarse. Entonces, durante estos momentos iniciales, buena parte del interés del profesional debería recaer en saber qué es lo que le impide venir a la sesión presencialmente y el trabajo terapéutico incorporará el objetivo de conseguir que el paciente pueda llegar a venir en persona al despacho. Caso bien distinto es el de si, en un tratamiento ya iniciado, el paciente decide abandonar la ciudad o el país pero no quiere dejar el tratamiento, no quiere o no puede desplazarse hasta el despacho y no accede a proseguir con otro terapeuta en su nuevo lugar de residencia, porque considera que el trabajo realizado puede seguirse mediante internet; entonces parecería que la mejor indicación para resolver la circunstancia planteada, sería el formato on line. Es evidente que existen multitud de casos y situaciones que apuntan a la indicación de una terapia on line, pero nuestra experiencia es la de que no existe una indicación "de elección" on line, sino que el formato on line puede ayudar en buena medida a reparar una situación terapéutica que, de no existir este formato, acabaría con una más que probable rotura definitiva del proceso terapéutico.

A menudo se ha caricaturizado el psicoanálisis por su rigidez metódica y por su poca flexibilidad a la hora de modificar aspectos del setting. En los últimos años ha tenido especial impacto lo que conocemos como psicoanálisis relacional, en el que por ejemplo se propone, aplica y defiende la posibilidad de que el analista o terapeuta se desplace hasta el domicilio del paciente si éste no está en condiciones de salir o, por el contrario, si interesa ver cómo se desarrolla su dinámica familiar in situ. Nos parece que esta opción corrige, en cierta medida, el carácter supuestamente rígido de las terapias psicoanalíticas pero advertimos, sin embargo, del riesgo que supone apresurarse en el afán de modernización, de flexibilización a la hora de crear nuevas herramientas que permitan llegar a más pacientes, ya sea por la distancia física o por sus mismas condiciones personales. En otras palabras, conviene no sucumbir ante el "fear of missing out" (FoMO), en el miedo a perderse algo (Przybylsky, Murayama, DeHaan y Gladwell, 2013). Deberíamos estar dispuestos a pensar si realmente esta herramienta ayuda a un paciente determinado, si supone un beneficio para él en el marco del tratamiento o si responde al intento de no "perder" un paciente a causa de la interrupción o de la derivación a otro profesional. Podemos hacer sesiones on line con casi cualquier paciente, pero no todos los pacientes están indicados para hacer una psicoterapia on line y la decisión es, como mínimo, tan importante como la que tiene lugar cuando valoramos la indicación de realizar las sesiones cara a cara o en el diván. Conviene estar atentos al riesgo de banalizar la relación terapéutica convirtiéndola en una sucesión de contactos superficiales, alejada del objetivo transformador que le es propio y que debería caracterizarla siempre, con independencia de las modalidades técnicas utilizadas. Probablemente el riesgo no está en la 
naturaleza misma del propio instrumento sino en la del terapeuta que, a veces de forma automática y poco reflexiva, se siente empujado a probar, a cambiar, a acceder a novedades que le hagan sentir que "no se queda fuera". El trato humano en vivo y en directo no es equiparable al on line. En ocasiones puede ser un buen recurso, e incluso puede ser un buen complemento, pero en ningún caso puede ser mejor y más real que el presencial.

\section{REFERENCIAS}

Andersson, G.; Carlbring, P.; Berger, T.; Almlöv, J.; Cuijpers, P. (2009). What Makes Internet Therapy Work? Cognitive Behaviour Therapy; 38(1), pp. 55-60.

Baudrillard, J. (2005). Cultura y simulacro. ( $7^{\mathrm{a}}$ ed.). Barcelona: Editorial Kairós.

Bauman, Z. (2005). Amor líquido. Acerca de la fragilidad de los vínculos humanos. Madrid: Fondo de Cultura Económica.

Botella, C.; Baños, R.; García Palacios, A.; Quero, S.; Guillén, V.; Pons, A. (2007). La utilización de las nuevas tecnologías de la información y la comunicación en psicología clínica. UOC papers, Vol. 4, pp. 32-41.

Brown, F. W. (1998). Rural telepsychiatry. Psychiatric Services, Vol. 49, pp. 963-964.

Castelnuovo, G.; Gaggioli, A.; Mantovani, F.; Riva, G. (2003). From Psychotherapy to e-therapy: The Integration of Traditional Techniques and New Communication Tools in Clinical Settings. Cyberpsychology and Behavior, Vol. 6(4), pp. 375-38.

Cestero-Mancera, A. M. (2006) "La comunicación no verbal y el estudio de su incidencia en fenómenos discursivos como la ironía". ELUA. Estudios de Lingüística. Vol. 20 (2006). ISSN $0212-$ 7636, pp. 57-77.

Cook, J. E.; Doyle, C. (2002). Working Alliance in Online Therapy as Compared to Face-to-Face Therapy: Preliminary Results. CyberPsychology \& Behavior, Vol. 5(2), pp. 95-105.

Encabo, E.; López, A. (2004). Diferencias de género y comunicaciónaspectos no verbales y propuestas didácticas. Didáctica (Lengua y literatura), ISSN 1130-0531, Vol. 16, 2004, pp. 45-56.

Griffits, F.; Lindemnmeyer, A.; Powell, J. et al. (2006). Why are health care interventions delivered over the Internet? A systematic review of the published literature. Journal of Medical Internet Research. Vol. 8, 2, pp. 10. Recuperado en enero 2007, en http://www.rider.edu/users/suler/psycyber/psycyber.html

Hanley, T. (2009). The working alliance in online therapy with young people: the preliminary indings. British Journal of Guidance \& Counselling, Vol. 3(37), pp. 257-269.

Hanley, T.; Reynolds, D. J. (2009). Counselling Psychology and the Internet: A review of the quantitative research into online outcomes and alliances within text-based therapy. Counselling Psychology Review, Vol. 24(2), pp. 4-13. 
King, R.; Bambling, M.; Lloyd, C.; Reid, W.; Thomas, I. (2006). Telephone and online counseling for young people: A naturalistic comparison of session outcome, session impact and therapeutic alliance. Counselling and Psychotherapy Research, Vol. 6(3), pp. 175-181.

Knaevelsrud, C.; Maercker, A. (2006). Does the Quality of the Working Alliance Predict Treatment Outcome in Online Psychotherapy for Traumatized Patients?. Journal of Medical Internet Research, Vol. 8(4).

Nickelson, D. (1998). Telehealth and the envolving health care system: strategic opportunities for professional psychology. Professional Psychology: Research and Practice, Vol. 29, pp. 527-535.

Núñez, M. ${ }^{a}$ P. (2000). Un aspecto básico para la didáctica de la lengua oral: el papel del lenguaje en la comunicación didáctica. Lenguaje y Textos, Vol. 16, pp. 155-172.

Pergament, D. (1998). Internet Psychotherapy: Current Status and Future Regulations. Health Matrix: Journal of Law-Medicine, Vol. 8(2).

Poyatos, F. (2003). La comunicación no verbal: Alguna de sus perspectivas de estudio e investigación. Revista de Investigación Lingüística. Vol. 6 (2).

Przybylski, A. K.; Murayama, K.; DeHaan, C. R.; Gladwell, V. (2013). Motivational, emocional, and behavorial correlatos of fear of Messing out. Computers in Human Behavior. Vol. 29, Issue 3, pp. $1841-1848$.

Ramón, R.; Segura, M. P.; Palanca, M. M.; Román, P. (2012). Habilidades sociales en enfermería. El papel de la comunicación centrado en el familiar. Revista Española de Comunicación en Salud, ISSN-e 1989-9882, Vol. 3(1), pp. 49-61.

Reynolds, D. J.; Stiles, W. B.; Grohol J. M. (2006). An investigation of session impact and alliance in internet based psychotherapy: Preliminary results. Counselling and Psychotherapy Research, Vol. 6(3), pp. 164-168.

Ritterband, L.; Gonder-Frederick, L.; Cox, D. et al. (2003). Internet interventions: in review, in use and into the future. Professional Psychology : Research and Practice, Vol. 34(5), pp. 527-534.

Rochlen, A. B.; Zack, J. S.; Speyer, C. (2004). Online Therapy: Review of Relevant Deinitions, Debates, and Current Empirical Support. Journal of Clinical Psychology, Vol. 6o(3), pp. 269-283.

Schultze, N. (2006). Success Factors in Internet-based Psychological Counseling. CyberPsychology \& Behavior, Vol. 9(5), pp. 623-626.

Suler, J. R. (2000). Psychotherapy in Cyberspace: A 5-Dimensional Model of Online and ComputerMediated Psychotherapy. CyberPsychology \& Behavior, Vol. 3(2), pp. 151-159.

Tate, D. F.; Zabinski, M. F. (2004). Computer and Internet Applications for Psychological Treatment: Update for Clinicians. Journal of Clinical Psychology, Vol. 6o(2), pp. 209-220.

Trujillo Borrego, A. (2005). Nuevas tecnologías y Psicología. Una perspectiva actual. Apuntes de Psicología, Vol. 23(3), pp. 321-335.

Tusón, A. (2002). El análisis de la conversación: entre la estructura y el sentido. Universitat Autònoma de Barcelona. Copyright (๑) Estudios de Sociolingüística. Vol. 3(1), 2002, pp. 133-153.

Vaimberg, R. (2012). Bases de la psicoterapia e intervenciones online. Intercanvis, Vol. 28. 
Vaimberg, R. (2014). Del diván y del escenario a la pantalla. PsiNotes.

Valdés, N.; Krause, M.; Álamo, N. (2011). ¿Qué Dicen y Cómo lo Dicen? Análisis de la comunicación verbal de pacientes y terapeutas en episodios de cambio. Revista argentina de clínica psicológica, ISSN 0327-6716, Vol. 20(1), pp. 15-28.

Vallejo, M. A.; Jordán, C. M. (2007). Psicoterapia a través de Internet. Boletín de Psicología, Vol. 91, pp. 27-42.

Wright, J. (2002). Online counselling: learning from writing therapy. British Journal of Guidance \& Counselling, Vol. 30(3), pp. 285-298.

Original recibido con fecha: 12/5/2016 Revisado: 13/5/2016 Aceptado: 30/6/2016 